\title{
A segurança do enfermeiro no atendimento pré-hospitalar ao paciente com quadro suspeito ou confirmado de COVID-19: Revisão integrativa
}

\author{
Nurse safety in pre-hospital care for patients with suspected or confirmed COVID-19: An \\ integrative review \\ Seguridad de las enfermeras en la atención prehospitalaria para pacientes con COVID-19 \\ sospechado o confirmado: Una revisión integradora
}

Recebido: 08/06/2021 | Revisado: 16/06/2021 | Aceito: 20/06/2021 | Publicado: 04/07/2021

\author{
Lívia Benevides Pinto Ravaglia de Aguiar \\ ORCID: https://orcid.org/0000-0001-9963-7105 \\ Centro Universitário Celso Lisboa, Brasil \\ E-mail: ravagliaaguiar@gmail.com \\ Thayná Anselmo de Araújo Cunha \\ ORCID: https://orcid.org/0000-0001-6227-331X \\ Instituto Brasil de Ensino, Brasil \\ E-mail: thaaycunha@hotmail.com
}

\begin{abstract}
Resumo
O estudo visa fomentar a educação continuada dos profissionais inseridos no Atendimento Pré-Hospitalar de casos suspeitos ou confirmados de COVID-19, reforçando os cuidados necessários neste contexto. Trata-se de uma revisão integrativa, cujo levantamento de dados se deu na Biblioteca Virtual de Saúde (BVS), onde encontram-se disponíveis dados da LILACS, SciELO, Bdenf, Medline entre outros. Foram selecionadas 06 publicações entre o período de 2019 a 2021. Após a análise dos dados coletados, aplicando-se os critérios de inclusão e exclusão, emergiram-se as categorias: A segurança do profissional de saúde e Segurança do paciente. Observou-se que o conhecimento e a assistência qualificada maximizam os possíveis resultados ao enfermo e garantem a segurança do profissional durante todo o processo de cuidado ao paciente. Além disto, a aplicação correta das medidas de proteção reflete na contenção da propagação do coronavírus.
\end{abstract}

Palavras-chave: Atendimento pré-hospitalar; COVID-19; Enfermeiro; Segurança.

\begin{abstract}
The study aims to promote the continuing education of professionals working in the Pre-Hospital Care of suspected or confirmed cases of COVID-19, reinforcing the necessary care in this context. This is an integrative review, whose data collection took place in the Virtual Health Library (VHL), where data from LILACS, SciELO, Bdenf, Medline, among others, are available. Six publications were selected from 2019 to 2021 . After analyzing the data collected, applying the inclusion and exclusion criteria, the following categories emerged: Health professional safety and Patient safety. It was observed that knowledge and qualified assistance maximize the possible results for the patient and ensure the professional's safety throughout the patient care process. In addition, the correct application of protection measures reflects in the containment of the coronavirus propagation.
\end{abstract}

Keywords: Pre-hospital care; COVID-19; Nurse; Safety.

\section{Resumen}

El estudio tiene como objetivo promover la formación continua de los profesionales que trabajan en la Atención Prehospitalaria de casos sospechosos o confirmados de COVID-19, reforzando la atención necesaria en este contexto. Se trata de una revisión integradora, cuya recolección de datos se realizó en la Biblioteca Virtual en Salud (BVS), donde se encuentran disponibles datos de LILACS, SciELO, Bdenf, Medline, entre otros. Se seleccionaron seis publicaciones de 2019 a 2021. Tras analizar los datos recogidos, aplicando los criterios de inclusión y exclusión, surgieron las siguientes categorías: Seguridad del profesional sanitario y Seguridad del paciente. Se observó que el conocimiento y la asistencia calificada maximizan los posibles resultados para el paciente y garantizan la seguridad del profesional durante todo el proceso de atención al paciente. Además, la correcta aplicación de las medidas de protección se refleja en la contención de la propagación del coronavírus.

Palabras clave: Atención prehospitalaria; COVID-19; Enfermero; Seguridad. 


\section{Introdução}

Com o uso cada vez mais frequente das mídias sociais, é possível notar que a sociedade tem tido o Atendimento PréHospitalar (APH) como objeto de atenção. Em decorrência da pandemia por COVID-19 nota-se um maior destaque aos profissionais que atuam em tal ambiente. Além do olhar mais atento da sociedade, a pandemia culminou em uma maior preocupação governamental no Brasil a respeito da organização de saúde e seus fluxos. É possível então afirmar que o contexto de saúde atual desperta um maior interesse e cuidado pela saúde daqueles que atuam na promoção da mesma (Ramos \& Sanna, 2005).

A respeito do APH, o Ministério da Saúde, por meio da Portaria no 1863/GM de 29 de setembro de 2003 (Ministério da Saúde, 2003), o define como a assistência prestada em um primeiro nível de atenção aos indivíduos com quadros agudos, de natureza clínica, traumática ou psiquiátrica, fora do ambiente hospitalar, que pode ou não acarretar sequelas ou evoluir a óbito.

Já no que tange o novo coronavírus (SARS-CoV-2), o Ministério da Saúde (2021) define a COVID-19 como uma infecção respiratória aguda que pode variar de casos assintomáticos e manifestações clínicas leves, até quadros moderados e graves que necessitem de um olhar mais atento em vista da rápida evolução que a doença pode ter. Este vírus pode ser transmitido de pessoa a pessoa por meio de pequenas gotículas do nariz ou da boca, ou através do contato com objetos e superfícies contaminadas seguido do contato em olhos, nariz ou boca.

O profissional de saúde, ao realizar procedimentos que gerem alto risco de aerossolização de secreções respiratórias, deve instituir as técnicas de precaução para aerossóis, fazendo uso dos equipamentos de proteção individual (EPI) específicos para este tipo de precaução. Neste caso, utiliza-se uma máscara de proteção respiratória com eficácia mínima na filtração de 95\% de partículas de até 0,3u. São estas as máscaras do tipo N95, N99, N100, PFF2 ou PFF3 (TelessaúdeRS, 2020).

No que se refere ao paciente, deve-se evitar o transporte interinstitucional de casos suspeitos ou confirmados, bem como a realização de exames de imagem que não alteram o curso da doença. A regulação avalia o cenário da solicitação do atendimento e a possibilidade de realizar procedimentos invasivos quando há suspeita de Covid-19. Cada caso possui sua particularidade, e a regulação informa à equipe da ambulância a necessidade do uso de EPI em maior proteção (Bahia, 2019).

Diante do exposto, o presente estudo tem por propósito fomentar a educação continuada dos profissionais inseridos no Atendimento Pré-Hospitalar de casos suspeitos ou confirmados de COVID-19 e reforçar os cuidados necessários neste ambiente para reduzir o risco de transmissão do coronavírus, tendo por base evidências técnicas e científicas.

\section{O Desenvolvimento do Atendimento Pré-Hospitalar no Brasil}

Nota-se, ao longo da história, a participação da enfermagem no socorro, atendimento inicial e resgate de doentes e feridos de guerra. Romanzini e Bock (2010) afirmam que na sociedade moderna uma guerra não declarada foi a principal responsável pela mortalidade em situações de urgência e emergência.

O APH surgiu no Brasil na cidade do Rio de Janeiro, em 1893, como uma medida de intervenção do Estado, que tinha por objetivo proporcionar um atendimento precoce, rápido e com transporte adequado a um serviço emergencial definitivo, a fim de aumentar a sobrevida dos pacientes (Martins \& Prado, 2003).

Em 21 de Maio de 2012 o Ministério da Saúde implantou um modelo de APH denominado Serviço de Atendimento Móvel de Urgência (SAMU). Este modelo tem por referência um estilo de atendimento francês, tendo sua equipe composta por médicos especialistas na área de emergência, e o modelo americano que inclui os técnicos em emergências médicas no nível básico, intermediário e paramédicos (Martins \& Prado, 2003) Assim, este modelo de atendimento visa unificar a estrutura de saúde e melhorar a assistência de saúde prestada (Ramos \& Sanna, 2005).

Para Romanzini e Bock (2010) "o APH compreende as ações iniciais realizadas em curto espaço de tempo pela equipe de resgate no local onde ocorre o agravo à saúde, seja ele traumático, clínico ou psíquico". A remoção segura das vítimas até 
um centro de atendimento hospitalar de referência é indispensável. Em vista do cenário, o enfermeiro encontra diversos desafios quanto à operacionalização do serviço.

Trazendo para a atualidade, a pandemia pelo coronavírus torna fundamental a reflexão sobre quais cuidados de enfermagem são necessários na preservação da segurança daqueles que atuam no atendimento de casos suspeitos ou confirmados de COVID-19 (Faro et al., 2020).

O profissional enfermeiro vem desenvolvendo e aprimorando atendimentos humanizados, aliados a técnicas de comprovação cientifica acerca de tal enfermidade. Aquino et al. (2020) recomendam a redução do contato social o que, consequentemente, diminui as chances de transmissão do coronavírus - que possui alta transmissibilidade se comparado a outros coronavírus do passado.

A fim de garantir maior segurança aos profissionais e pacientes, o Ministério da Saúde (2020) instituiu algumas mudanças nos procedimentos de rotina dos casos suspeitos ou confirmados de infecção por COVID-19. A exemplo, durante o transporte de pacientes que se enquadram nos critérios supracitados, mantêm-se as janelas abertas e o exaustor das ambulâncias ligados. Isto promove uma melhor ventilação interna do veículo.

\section{A Atuação do Enfermeiro no APH}

A inserção do enfermeiro no APH móvel se deu através da Política Nacional de Atenção às Urgências, que possui diversas categorias quanto a composição da equipe (Adão \& Santos, 2012). Um dos tipos de atendimento que mais se destaca é o Suporte Avançado de Vida, onde a ambulância é composta por motorista socorrista, médico e enfermeiro.

O enfermeiro atua em conjunto com a equipe de APH móvel em diversos ambientes, com restrição de espaço físico e em situações limite de tempo da vítima e cena. Para Rodrigues (2017) essas situações evidenciam a necessidade de decisões imediatas, tendo por base protocolos, conhecimento e rápida avaliação.

O médico, o socorrista e o enfermeiro são responsáveis, em conjunto, pela assistência. Uma das metas da assistência é a estabilização do paciente, seja no local da ocorrência ou durante o transporte até um local de atendimento fixo (Adão \& Santos, 2012).

No APH móvel público o profissional enfermeiro atua de acordo com a avaliação das necessidades da vítima, definição de prioridades, intervenções necessárias, e por meio da reavaliação contínua durante todo o período de cuidado do paciente. Isto torna possível um entendimento da significância deste profissional neste tipo de atendimento.

\section{Metodologia}

O estudo baseia-se em uma revisão integrativa da literatura, que segundo Mendes et al. (2008) tem por finalidade reunir e resumir o conhecimento científico produzido a respeito da temática abordada, abreviando as evidências disponíveis. Para Souza et al. (2010) uma revisão integrativa dá-se ainda por meio de uma ampla abordagem metodológica e amostra em conjunto com a multiplicidade de propostas. A revisão integrativa permite a compreensão de estudos, conceitos complexos, teorias e problemas.

Broome (2000) divide a revisão integrativa em seis fases, permitindo um conhecimento uniforme e prestando um suporte para a tomada de decisão. Na elaboração do presente estudo, utilizou-se quatro fases, que são descritas na Figura 1. 
Figura 1 - As fases da revisão integrativa.

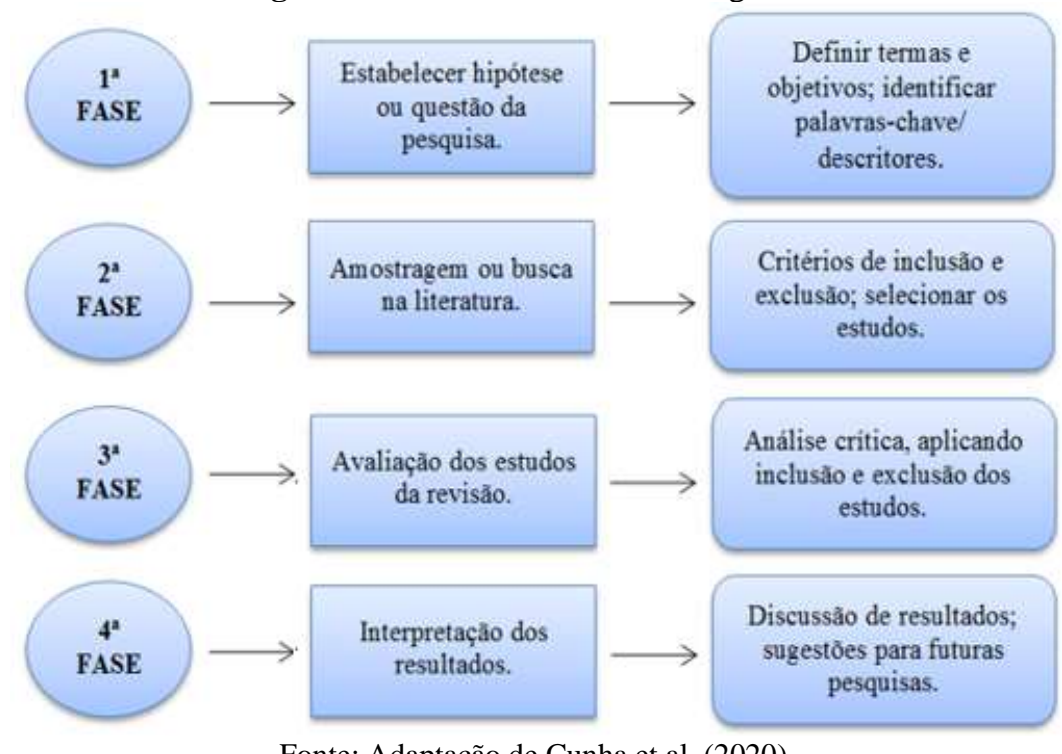

Fonte: Adaptação de Cunha et al. (2020).

$\mathrm{Na}$ primeira etapa identifica-se a questão norteadora da pesquisa por meio da estratégia PI, onde $\mathrm{P}$ se refere à população abordada e I ao aspecto de interesse do estudo. Formulou-se, então, a seguinte pergunta: Como garantir a segurança do profissional de saúde frente ao APH de casos suspeitos ou confirmados de COVID-19?

A segunda etapa se deu por meio da busca de literatura através da base de dados da Biblioteca Virtual em Saúde, na qual se encontram dados da Literatura Latino-Americana e do Caribe em Ciências da Saúde, Scientific Eletronic Library Online, Base de Dados de Enfermagem, Medical Literature Analysis and Retrievel System Online, entre outros. Empregaramse as palavras-chave "atendimento pré-hospitalar", "COVID-19", "enfermeiro" e "segurança", com o intuito de abranger a pesquisa por meio de títulos, resumos e assuntos. Aplicou-se ainda o operador booleano "AND" para realizar a combinação das palavras e equações.

Quando aplicada as palavras-chave "atendimento pré-hospitalar" AND "COVID-19", foram identificados 359 estudos. Ao se aplicarem os filtros com disponibilidade na íntegra, idioma e recorte temporal estabelecidos como critérios de inclusão, foram encontrados 14 estudos. Seguindo a leitura de títulos e resumos, verificou-se que somente dois estudos contemplavam o assunto de interesse.

No segundo cruzamento, utilizaram-se as palavras-chave "COVID-19" AND "enfermeiro", sendo encontradas 379 publicações. Após aplicação dos filtros, realizou-se a leitura prévia do título e resumo de 52 destas publicações. Diante disto, foi possível verificar que apenas três estudos contemplavam a temática de interesse.

Por fim, foi aplicado um terceiro cruzamento, utilizando as palavras-chave "COVID-19" AND "enfermeiro" AND "segurança", resultando em 27 publicações. Das publicações encontradas, após aplicação dos filtros, foi realizada a leitura prévia do resumo de 12 destas. Identificou-se que dois estudos contemplavam o assunto de interesse, contudo um destes já havia sido selecionado em cruzamento anterior. Deste modo, um estudo foi selecionado.

Ao todo, foram encontrados 765 estudos, dos quais somente 78 estavam de acordo com os critérios de inclusão, porém, destes, 72 não abordavam o tema de interesse. Foram então selecionados seis estudos. Estabeleceram-se ainda como critérios de inclusão: materiais disponíveis em texto completo, em português e com recorte temporal de dois anos (2019 2021); como critérios de exclusão: artigos editoriais.

$\mathrm{Na}$ terceira e quarta fase, analisando e interpretando os resultados obtidos, notou-se que os estudos destacam que a assistência comprovadamente qualificada e treinada reduz a transmissibilidade da COVID-19, evitando a contaminação dos profissionais de saúde que prestam atendimento ao paciente infectado, maximizando a qualidade de atendimento prestado, 
possíveis resultados ao enfermo e garante ainda a segurança do profissional que presta cuidados ao paciente. Após a análise dos resultados obtidos, emergiram-se as seguintes categorias: "A segurança do profissional de saúde" e "Segurança do paciente".

\section{Resultados}

Compreendendo mais sobre a temática em questão, ao final das buscas realizadas, foram selecionados seis artigos descritos no Quadro 1.

Quadro 1 - Caracterização dos Artigos da Amostra. Rio de Janeiro, Brasil, 2021.

\begin{tabular}{|c|c|c|c|}
\hline Equação de busca & Autor & Ano & Título \\
\hline Por descritores & & & \\
\hline $\begin{array}{l}\text { "Atendimento Pré- } \\
\text { Hospitalar" AND } \\
\text { "COVID-19" }\end{array}$ & Bahia & 2020 & $\begin{array}{l}\text { Nota Técnica COE Saúde n. 58, de } 14 \text { de abril } \\
\text { de } 2020 \text { - Orientações para o serviço de } \\
\text { atendimento pré-hospitalar móvel quanto às } \\
\text { instruções do uso de Equipamentos de } \\
\text { Proteção Individual EPI - durante a } \\
\text { assistência aos casos suspeitos ou } \\
\text { confirmados de infecção pelo novo } \\
\text { Coronavírus (SARS-COVID-19). }\end{array}$ \\
\hline $\begin{array}{l}\text { "Atendimento Pré- } \\
\text { Hospitalar" AND }\end{array}$ & & & $\begin{array}{l}\text { Nota Técnica 009/2020 - Recomendações e } \\
\text { medidas para os profissionais do SAMU que }\end{array}$ \\
\hline “COVID-19" & Florianópolis & 2020 & $\begin{array}{l}\text { tiveram contato com pessoas que } \\
\text { apresentaram teste para COVID-19 com } \\
\text { resultado positivo. }\end{array}$ \\
\hline $\begin{array}{l}\text { "COVID-19" AND } \\
\text { "Enfermeiro" }\end{array}$ & Verbeek et al. & 2020 & $\begin{array}{l}\text { Personal protective equipment for preventing } \\
\text { highly infectious diseases due to exposure to } \\
\text { contaminated body fluids in healthcare staff. }\end{array}$ \\
\hline $\begin{array}{l}\text { "COVID-19" AND } \\
\text { "Enfermeiro" }\end{array}$ & $\begin{array}{l}\text { Busanello et } \\
\text { al. }\end{array}$ & 2021 & $\begin{array}{l}\text { Boas práticas para aspiração de vias aéreas } \\
\text { de pacientes em terapia intensiva. }\end{array}$ \\
\hline $\begin{array}{l}\text { "COVID-19" AND } \\
\text { "Enfermeiro" }\end{array}$ & Reis et al. & 2020 & $\begin{array}{l}\text { Atuação da enfermagem no cenário da } \\
\text { pandemia COVID-19. }\end{array}$ \\
\hline $\begin{array}{l}\text { "COVID-19" AND } \\
\text { "Enfermeiro" AND } \\
\text { "Segurança" }\end{array}$ & Marques et al. & 2020 & $\begin{array}{l}\text { COVID-19: Cuidados de enfermagem para } \\
\text { segurança no atendimento de serviço pré- } \\
\text { hospitalar móvel. }\end{array}$ \\
\hline
\end{tabular}

Fonte: Autoras.

Quanto ao tipo de pesquisa, constituíram a amostra notas técnicas (dois), estudo descritivo quantitativo (um); estudo descritivo exploratório (dois); estudo qualitativo exploratório (um).

\section{Discussão}

O material utilizado foi selecionado por meio do exame de artigos, com o intuito de facilitar o entendimento acerca da temática abordada e demonstrar a atuação do enfermeiro no atendimento intra e pré-hospitalar ao paciente com COVID-19.

Em situações de pandemia torna-se necessário instituir medidas que promovam maior segurança aos profissionais atuantes na linha de frente e pacientes diagnosticados com a doença pandêmica, tendo em vista o risco iminente de contágio pelo manuseio de materiais biológicos e produtos químicos, que aumentam a suscetibilidade de danos para a saúde. Diante disto, emergiram-se as categorias que seguem. 


\section{A segurança do profissional de saúde}

Para Marques et al. (2020) a segurança da equipe é primordial nos atendimentos do serviço pré-hospitalar móvel. O uso correto do EPI deve ser adotado por todos os profissionais. Entende-se que o manejo da pandemia por COVID-19 exige critérios mais específicos, visto que o cenário mundial gera um alerta quanto aos riscos de desabastecimento e superlotação dos serviços de saúde.

Os profissionais de saúde que tratam pacientes com infecções como o coronavírus se expõem constantemente ao risco de infecção. Assim, Marqus et. Al (2020) afirmam que a paramentação dos profissionais tem início logo após o acionamento da ambulância e para isto são disponibilizados EPIs específicos: máscara cirúrgica, máscara N95, máscaras face Shield, luvas de procedimento, toucas descartáveis e aventais cirúrgicos estéreis impermeáveis de manga longa (100\% polipropileno) e punho $100 \%$ algodão.

Por se tratar de APH móvel, identifica-se a necessidade de adequação às realidades enfrentadas nesse ambiente. Assim sendo, Marques et al. (2020) afirmam que o avental disponibilizado e preconizado pelo Ministério da Saúde gera sensação de insegurança no contexto pré-hospitalar, uma vez que não permite a cobertura de toda a extensão corporal, o que se agrava nos profissionais que possuem maior estatura.

De acordo com Verbeek et al. (2020), a Associação Brasileira de Medicina de Emergência e algumas outras instituições sugerem, desde 1996, que os profissionais do APH utilizem macacão com proteção da cabeça (proteção $360^{\circ}$ ), confeccionado com polietileno de alta densidade, com punhos e tornozelos de elástico - a exemplo, o Tyvec/tychen. Essa medida tem por objetivo ampliar a proteção destes profissionais e fazer com que eles possam se sentir mais seguros e confortáveis na realização de suas atividades laborais. Além disto, os autores reforçam que alguns EPI foram adaptados para que haja uma maior facilidade na sua colocação, uso e remoção.

A fim de preservar a saúde e segurança do profissional, após o atendimento os óculos e protetores faciais são higienizados com água e sabão e, quando secos, são submetidos à fricção com álcool a 70\%. Já as botas são higienizadas com borrifação de hipoclorito de sódio a 1\%, seguida de friç̧ão com pano úmido. Cabe ressaltar a necessidade do uso rotineiro do macacão de serviço com mangas longas (Fundação Casa, 2020).

Não menos importante, outra medida fortemente adotada pela sociedade como um todo é a higienização das mãos. Reis et al. (2020) alegam que esta é a medida de prevenção mais adequada contra o novo coronavírus - além do uso de EPI. O Ministério da Saúde (2020) traz que as mãos devem ser lavadas obrigatoriamente entre os atendimentos a diferentes pacientes, após a finalização de cada atendimento e antes de retirada de máscaras e óculos. Diante da impossibilidade de higienizá-las com água e sabão, faz-se o uso de preparações alcoólicas a 70\%. A Agência Nacional de Vigilância Sanitária (2012), por meio da $\operatorname{RDC} n^{\circ} 15$, reforça tais orientações e ainda reforça que aplicar grande quantidade do produto, seja o sabão ou a preparação alcoólica, sem realizar a fricção não há o efeito esperado.

Pensando na segurança profissional, a Secretaria de Estado da Saúde de Florianópolis elaborou a Nota Técnica nº 009, de 18 de junho de 2020 (Florianópolis, 2020), em que existem recomendações e medidas para os profissionais do SAMU que tiveram contato com casos COVID confirmados.

Considerando que a equipe de enfermagem representa o grande contingente de recursos humanos na atenção à saúde e, em grande parte das vezes, é a responsável direta pela assistência, cabe refletir a relevância da adoção dessas medidas de segurança e seus espelhos na atuação dos enfermeiros que prestam atendimento especialmente em casos suspeitos ou confirmados de COVID-19. 


\section{Segurança do paciente}

No que corresponde a segurança do paciente, o Ministério da Saúde (2013), por meio da Portaria n ${ }^{\circ} 529$ de 01 de abril de 2013, a define como "redução, a um mínimo aceitável, do risco de dano desnecessário associado ao cuidado de saúde" (artigo 4, alínea I). Deste modo, vale ressaltar que a segurança do paciente é de grande importância no que tange a qualidade do cuidado e depende da oferta de uma assistência segura.

Ao chegar no local de atendimento/transporte, o profissional médico e o enfermeiro avaliam a clínica do paciente, verificando se há alterações clássicas de quadro de COVID-19 como: quadro febril, tosse, coriza, taquicardia, taquidispneia ou queda de saturação em ar ambiente, avaliando também a necessidade de suplementação de oxigênio (Marques et al., 2020).

Já durante o transporte do paciente para uma unidade de referência, a equipe realiza a monitorização cardíaca e o acompanhamento dos parâmetros vitais. O Ministério da Saúde (2020), por meio do "Protocolo de Tratamento do Novo Coronavírus (2019-nCov)", preconiza que o profissional médico e o enfermeiro responsáveis por este monitoramento sejam capazes de reconhecer eventual desconforto respiratório e, se necessário, instituir máscara de oxigênio de alto fluxo. Se hou ver um quadro de desconforto respiratório persistente, fica a critério do profissional médico a realização da intubação orotraqueal e ventilação mecânica.

Conferir as bombas de infusão, ajustar $\mathrm{FiO} 2$ para 100\% e conectar o ventilador mecânico ao cilindro de oxigênio diretamente na válvula do próprio cilindro são alguns dos cuidados que se deve ter ao transportar um paciente que segue em ventilação mecânica (Busanello et al., 2021).

De acordo com Marques et. al (2020), o objetivo final do atendimento no transporte de um paciente suspeito ou confirmado de COVID-19 é garantir a sua segurança para uma unidade hospitalar de referência. Para isto, caso o paciente esteja em oxigenoterapia, a máscara cirúrgica deverá ser utilizada por cima da cânula de oxigênio.

Segundo a Nota Técnica COE Saúde $n^{\circ}$ 58, de 14 de abril de 2020 (Bahia, 2020), se houver necessidade de acompanhante, este também deverá fazer uso da máscara cirúrgica durante todo o trajeto. Se o mesmo espirrar ou tossir, deverá utilizar lenços de papel, seguido da higienização das mãos com álcool $70 \%$.

Todas as medidas de precaução são fundamentais neste momento crítico em vista dos inúmeros riscos pela exposição. Deste modo, quando o atendimento primário ou transporte intra-hospitalar é realizado, prioriza-se a qualidade do atendimento através de uma assistência capacitada e segura.

\section{Considerações Finais}

A maior inserção do profissional enfermeiro no âmbito do atendimento com suporte avançado ou básico de vida ampliou o espaço deste profissional no APH nos últimos anos. Nesse sentido, a garantia de segurança do profissional que presta atendimento é imprescindível durante todo o processo de assistência à saúde da população-alvo do APH, pois isto tem impacto direto na qualidade da assistência e na saúde do profissional.

Identifica-se por parte dos enfermeiros uma preocupação com esta segurança, visto que foram adotadas condutas para a prevenção e controle da pandemia mediante a utilização de equipamentos, materiais e preparo da ambulância. Assim sendo, aspectos subjetivos dos profissionais envolvidos devem ser considerados, como o preparo técnico e psicológico, que é fundamental tanto para o atendimento à população e segurança na exposição ao coronavírus.

Essa é uma prática que exige conhecimento aprimorado e contínuo, capacidade de lidar com situações de estresse e uma equipe de profissionais ampliada que difere da prática hospitalar. A pesquisa traz à tona ainda a necessidade de visibilidade dos cuidados em enfermagem, de modo que haja maior investimento nos profissionais, culminando em uma assistência cada vez mais eficiente e segura, reforçando nos atendimentos também os princípios de integralidade e resolutividade preconizados pelo SUS. 
O contexto decorrente da pandemia pelo novo coronavírus, com cepas ainda carentes de estudos comportamentais do vírus nos diversos ambientes e nas respostas à imunologia humana, um alerta foi disparado: o profissional que atua na linha de frente ao combate de doenças transmissíveis deve ser mais bem capacitado, atuar em ambiente com as condições de trabalho adequadas ao exercício de sua profissão e sua segurança, além de ser mais valorizado pela sociedade como um todo.

Por fim, os resultados obtidos pelo estudo comprovam o quão fundamental é a atualização profissional, de modo que esta não atenda apenas expectativas momentâneas, mas também se associe a capacidade de adaptação do profissional. Para tal, é preciso que mais estudos sejam realizados acerca da temática abordada. O conhecimento transforma e isso impacta diretamente na qualidade do atendimento prestado, bem como na segurança das pessoas inseridas no atendimento à saúde, sejam os profissionais de saúde ou os pacientes.

\section{Referências}

Adão, R. S., \& Santos, M. R. (2012). Atuação do enfermeiro no atendimento pré-hospitalar móvel. REME (Online), 16(4), 601-608. http://reme.org.br/artigo/detalhes/567

Agência Nacional de Vigilância Sanitária. (2012). Resolução RDC n. 15, de 15 de março de 2012. Dispõe sobre requisitos de boas práticas para o processamento de produtos para saúde e dá outras providências. Diário Oficial da União.

Aquino, E. M. L., Silveira, I. H., Pescarini, J. M., Aquino, R., Souza-Filho, J. A., Rocha, A. S., Ferreira, A., Victor, A., Teixeira, C., Machado, D. B., Paixão, E., Alves, F. J. O., Pilecco, F., Menezes, G., Gabrielli, L., Leite, L., Almeida, M. C. C., Ortelan, N., Fernandes, Q. H. R. F., ... Lima, R. T. R. S. (2020). Medidas de distanciamento social no controle da pandemia de COVID-19: Potenciais impactos e desafios no Brasil. Ciênc. saúde coletiva (Online), 25(suppl 1), 2423-2446. https://doi.org/10.1590/1413-81232020256.1.10502020

Bahia. (2019). Plano Estadual de Saúde da Bahia (PES): 2020-2023. Secretaria da Saúde. http://www.saude.ba.gov.br/wp-content/uploads/2020/10/PES2020-2023.pdf

Bahia. (2020). Nota Técnica COE Saúde n. 58, de 14 de abril de 2020. Orientações para o serviço de atendimento pré-hospitalar móvel quanto às instruções do uso de Equipamentos de Proteção Individual EPI - durante a assistência aos casos suspeitos ou confirmados de infecção pelo novo Coronavírus (SARSCOVID-19). Secretaria da Saúde. http://www.saude.ba.gov.br/wp-content/uploads/2020/05/NT_n_58_Sobre_profissionais_de_atendi mento_movel_pre_hospitalar.pdf

Broome, M. E. (2000). Integrative literature reviews for the development of concepts. In Rodgers, B. L., \& Knafl, K. A. (Eds.), Concept development in nursing: Foundations, techniques and applications. Saunders.

Busanello, J., Härter, J., Bittencourt, C. M., Cabral, T. S., \& Silveira, N. P. (2021). Boas práticas para aspiração de vias aéreas de pacientes em terapia intensiva. J. nurs. health., 11(1). https://periodicos.ufpel.edu.br/ojs2/index.php/enfermagem/article/view/19127/12525

Faro, A., Bahiano, M. A., Nakano, T. C., Reis, C., Silva, B. F. P., \& Vitti, L. S. (2020). COVID-19 e saúde mental: A emergência do cuidado. Estud. Psicol. (Campinas, Online), 37, Artigo e200074. https://doi.org/10.1590/1982-0275202037e200074

Florianópolis. Nota Técnica 009/2020. (2020). Recomendações e medidas para os profissionais do SAMU que tiveram contato com pessoas que apresentaram teste para COVID-19 com resultado positivo. Secretaria de Estado da Saúde. https://www.saude.sc.gov.br/coronavirus/arquivos/NOTATECNICA-009-2020SAMU-DAPM-SUE-SES.pdf

Fundação Casa. (2020). Caderno Técnico Superintendência de Saúde: Conceitos, diretrizes e procedimentos. https://fundacaocasa.sp.gov.br/wpcontent/uploads/2021/04/Caderno_Superintendencia_de_Saude.pdf

Marques, L. C., Lucca, D. C., Alves, E. O., Fernandes, G. C. M., \& Nascimento, K. C. (2020). COVID-19: Cuidados de enfermagem para segurança no atendimento de serviço pré-hospitalar móvel. Texto \& contexto enferm. (Online), 29, Artigo e20200119. https://doi.org/10.1590/1980-265X-TCE-2020-0119

Martins, P. P. S., \& Prado, M. L. (2003). Enfermagem e serviço de atendimento pré-hospitalar: Descaminhos e perspectivas. Rev. bras. enferm. (Online), 56(1), 71-75. https://doi.org/10.1590/S0034-71672003000100015

Mendes, K. D. S., Silveira, R. C. C. P., \& Galvão, C. M. (2008). Revisão integrativa: Método de pesquisa para a incorporação de evidências na saúde e na enfermagem. Texto \& contexto enferm. (Online), 17(4), 758-764. https://doi.org/10.1590/S0104-07072008000400018

Ministério da Saúde. (2003). Portaria n. 1863, de 29 de setembro de 2003. Institui a Política Nacional de Atenção às Urgências, a ser implantada em todas as unidades federadas, respeitadas as competências das três esferas de gestão. Diário Oficial da União.

Ministério da Saúde. (2012). Portaria n. 1.010, de 21 de maio de 2012. Redefine as diretrizes para a implantação do Serviço de Atendimento Móvel de Urgência (SAMU 192) e sua Central de Regulação das Urgências, componente da Rede de Atenção às Urgências. Diário Oficial da União.

Ministério da Saúde. (2013). Portaria n. 529, de $1^{\text { }}$ de abril de 2013. Institui o Programa Nacional de Segurança do Paciente (PNSP). Diário Oficial da União.

Ministério da Saúde. (2020). Protocolo de Tratamento do Novo Coronavírus (2019-nCoV). http://biblioteca.cofen.gov.br/wpcontent/uploads/2020/02/Protocolo-de-Tratamento-do-Novo-Coronav\%C3\%ADrus.pdf 
Research, Society and Development, v. 10, n. 8, e5010816976, 2021

(CC BY 4.0) | ISSN 2525-3409 | DOI: http://dx.doi.org/10.33448/rsd-v10i8.16976

Ministério da Saúde. (2021). Guia de vigilância epidemiológica: Emergência de saúde pública de importância nacional pela doença pelo coronavírus 2019 Covid-19: Versão $\quad 3 . \quad$ https://www.conasems.org.br/wp-content/uploads/2021/03/Guia-de-vigila\%CC\%82ncia-epidemiolo\%CC\%81 gica-dacovid_19_15.03_2021.pdf

Ramos, V. O., \& Sanna, M. C. (2005). A inserção da enfermeira no atendimento pré-hospitalar: Histórico e perspectivas atuais. Rev. bras. enferm. (Online), 58(3), 355-360. https://doi.org/10.1590/S0034-71672005000300020

Reis, L. M., Lago, P. N., Carvalho, A. H. S., Nobre, V. N. N., \& Guimarães, A. P. R. (2020). Atuação da enfermagem no cenário da pandemia COVID-19. Nursing (São Paulo), 23(269), 4765-4772. https://doi.org/10.36489/nursing.2020v23i269p4765-4772

Rodrigues, M. V. (2017). Atuação do enfermeiro no atendimento pré-hospitalar na realidade brasileira: Revisão integrativa. [Trabalho de Término de Curso, Graduação em Enfermagem, Faculdade de Ciências da Saúde do Trairi]. Repositório Científico da Universidade Federal do Rio Grande do Norte. https://monografias.ufrn.br/jspui/handle/123456789/4265

Romanzini, E. M., \& Bock, L. S. (2010). Conceptions and feelings of nurses working in emergency medical services about their professional practice and training. Rev. latinoam. enferm. (Online), 18(2), 105-112. https://doi.org/10.1590/S0104-11692010000200015

Souza, M. T., Silva, M. D., \& Carvalho, R. (2010). Integrative review: What is it? How to do it? Einstein (Sao Paulo. Online), 8(1), 102-106. https://doi.org/10.1590/S1679-45082010RW1134

TelessaúdeRS. (2020). Quais as máscaras devem ser utilizadas por profissionais de saúde? https://www.ufrgs.br/telessauders/post_coronavirus/quais-asmascaras-devem-ser-utilizadas-por-profissionais-de-saude/

Verbeek, J. H., Rajamaki, B., Ijaz, S., Sauni, R., Toomey, E., Blackwood, B., Tikka, C., Ruotsalainen, J.H, \& Kilinc Balci, F. S. (2020). Personal protective equipment for preventing highly infectious diseases due to exposure to contaminated body fluids in healthcare staff. Cochrane database syst. rev. (online), 4 , Article CD011621. https://doi.org/10.1002/14651858.cd011621.pub4 